\title{
An Evaluation of the Temperature Increase from PZT Micro-Transducers for Acoustic Trapping
}

\author{
Linda Johansson ${ }^{1}$, Mikael Nilsson ${ }^{2}$, Tobias Lilliehorn ${ }^{1}$, Monica Almqvist ${ }^{2}, J^{\prime}$ han Nilsson ${ }^{2}$, \\ Thomas Laurell ${ }^{2}$, Stefan Johansson ${ }^{1}$ \\ ${ }^{1}$ Department of Engineering Sciences, Uppsala University, Box 534, S-751 21 Uppsala, Sweden, SWEDEN and \\ ${ }^{2}$ Department of Electrical Measurements, Lund University, Box 118, S-221 00 Lund, SWEDEN
}

\begin{abstract}
This paper reports a comparison of soft and hard piezoceramic transducer materials used for ultrasonic standing wave particle trapping in a microfluidal bioanalytical platform. The investigation is made with the objective to obtain high acoustic forces with a minimum of temperature increase. Temperature is a critical parameter for bioassays and most often need to be kept below a certain level to allow handling of cells and proteins. The main conclusions in this paper are that it is possible to get efficient trapping with a temperature increase of only a few degrees and that a hard piezoceramic material has advantages in an application such as this.
\end{abstract}

\section{INTRODUCTION}

Several groups have reported the use of acoustic forces in fluidic systems for separation or trapping of particles and cells in macro-scale resonators, e.g [1]. Our group has developed a micro-scale system that uses higher frequencies and thus stronger trapping force [2]. The platform enables trapping at locally defined positions in a microfluidic device. The trapping positions are individually controlled and enable particles or cells passing by in a fluid to be trapped in a non-contact manner. The platform is very versatile and has shown great potential to be used in cellbased bioassays. Viability of yeast cells and neural stem cells during trapping has been proven "in press" [3].

A schematic view of the platform is shown in Fig. 1. Miniature multilayer PZT-ultrasound transducers are integrated in the bottom of a microchannel and are designed to work as acoustic resonators in thickness mode at a frequency of around $12 \mathrm{MHz}$. Properly matched channel height allows a standing wave to be formed in the fluid layer between transducers and a glass-reflector at the top of the channel. A local pressure minimum in the center of the channel defines the trapping site. Temperature rise in the channel is caused by mechanical and dielectric loss in the PZT transducers. The standard measurements of dielectric loss $(\tan \delta)$ and mechanical loss $\left(\mathrm{Q}^{-1}\right)$ are not obviously sufficient for predictions of which material is the most

The Swedish Foundation for Strategic Research is acknowledged for its financial support.

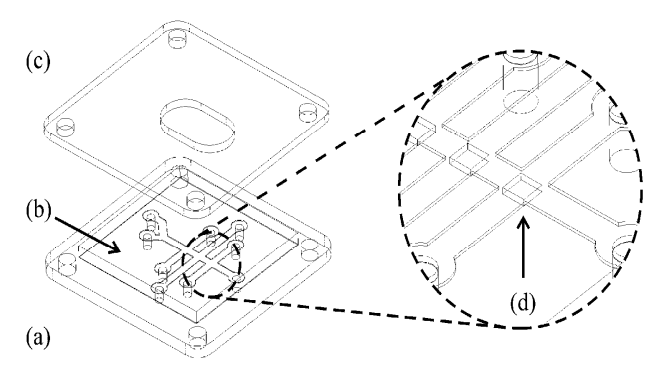

Figure 1. View of platform. The multilayered actuators measure $550 \mu \mathrm{m}$ in length and $200 \mu \mathrm{m}$ in height and are mounted in the bottom channel wall. Marked in the figure are transducer plate including electrical circuitry and actuators (a), channel structure (b), top brass plate (c) and a close-up of an actuator (d). Not shown in this figure is the lower brass plate which is added to ensure even pressurization.

favourable with respect to both temperature and trapping force for our miniature transducers. Data from manufacturers are based on a larger geometry with less dominant boundary conditions and also do not involve multilayer structures. To be able to select the optimal transducer material, the acoustic output and the fluid temperature is measured. The measurements will also give a quantitative value of temperature rise in the bioanalytical platform, and evaluate the fluorescent temperature measurement method for this application.

\section{EXPERIMENTAL}

Transducer plates (Fig. 1) are fabricated according to [2], generating a bottom channel surface that consists of multilayer transducers cast in epoxy (epotec 301-2). Wet etched silicon channel structures are anodically bonded to the reflector glass plate and aligned on top of the transducer plate. The platform is sealed by pressing the upper and lower brass plate together with screws. The measurements are performed in several steps. The impedance spectrums of the transducers are measured in air without channel structure and the resonance and anti-resonance frequency are determined. 
The acoustic pressure is measured with a burst sinusoidal signal at some chosen voltage levels using a miniature hydrophone of $0.2 \mathrm{~mm}$ diameter in a water bath. The hydrophone is aligned at a distance of 35 wavelengths from the transducer surface. This position is in the far field region and pressure measured here will differ from the pressure in the standing wave in the closed $\lambda / 2$ channel, but it can be used for comparison of the transducer materials. There are several reasons for measuring at longer distances. Difficulties separating electrical pulse from acoustic pulse and from multiple reflections from the hydrophone surface make it impossible to measure output pressure at distances close to the transducer. The effect of introducing an object such as the hydrophone into the acoustic field is prominent close to the transducer. Also by measuring in the far field, the result is much less sensitive to misalignment. To characterise the field, the square root of $P_{i}$ (time integral of the square of the instantaneous acoustic pressure in the pulse integrated over the whole pulse) is chosen since it shows good repeatability.

The piezoelectric materials (EDO EC-69 and EC-76) have different transducer properties and the drive voltage necessary to obtain the same acoustic pressure is calculated assuming that the pressure $P_{i}$ is linearly related to the voltage. The temperature in the fluid channel is thereafter measured, for continuous drive signal, by measuring the temperature sensitive fluorescent response in Rhodamine B $(0.1 \mathrm{mmol} / \mathrm{L}$ in deionised water $)$ in a fluorescence microscope [4]. Fluid flow is $0.5 \mu \mathrm{l} / \mathrm{min}$, which is lower than the flow usually used for trapping ( $4 \mu \mathrm{l} / \mathrm{min})$, and is obtained by use of a syringe pump. Between each measurement a fluid pulse was applied which served to wash away particles that may stick to surfaces. Two minutes of steady flow was required for fluid temperature to reach a steady state, which lasted over 10 minutes. After 2 minutes of steady flow, the microscope aperture was opened, the platform surface illuminated and a picture of the actuator surface was taken. For every measurement series, a reference picture with no acoustic field was used to define maximum intensity and was subtracted from every picture to account for background intensity and decrease in illumination source intensity. Exposure time was chosen to give max intensity for this white reference.

The preceding calibration step to relate fluorescence intensity to temperature was performed in a thermally controlled environment. Temperature of the whole platform was raised by heating the lower brass plate with a peltier element, while keeping the platform thermally isolated with polymer foam. Temperature was measured by a thermocouple (type $\mathrm{K}$ ) in contact with channel structure and when temperature had stabilised on a given level, fluorescence intensity in the fluid channel was recorded. Fluid flow was $0.5 \mu 1 / \mathrm{min}$.

\section{RESULTS AND DISCUSSION}

Typical impedance spectra of transducers in air is shown in Fig. 2 and the thickness resonance peaks are identified for series and parallel resonance for EC-76 (11.6 and $12.3 \mathrm{MHz}$, respectively) and EC-69 (12.0 and $12.3 \mathrm{MHz}$, respectively). The absolute level of impedance is much higher for EC-69 since this material has a lower dielectric constant.

Results from the hydrophone pressure measurements are shown in Fig. 3. For a given voltage level over the element, the piezoelectrically softer (EC-76) material delivers higher output pressure than the piezoelectrically harder (EC-69) material, with a factor of two or more. For EC-76, the difference between series resonance and parallel resonance is noticeable, while for EC-76 both resonances give similar output pressure. The output pressure is assumed to be linearly dependent on the applied voltage (fitted curves). Good trapping is known to be achieved at a drive signal of 7 $\mathrm{V}$ for the EC-76 transducers at series resonance. At this voltage level, the EC-76 parallel frequency gives $74 \%$ of the pressure needed for good trapping and EC-69 for series resonance and parallel resonance frequency gives about $37 \%$ of that pressure. With an Agilent function generator we are limited to a drive voltage of $10 \mathrm{~V}$ for a load of $50 \Omega$. Due to the high impedance of the EC-69 transducers the output pressure will be limited to about $3 \mathrm{Pas}^{1 / 2}$. At this pressure level, the voltage applied across the transducers for series and parallel resonance respectively is $5.0 \mathrm{~V}$ and $6.5 \mathrm{~V}$ for EC-76 and 13.5 V and 14.0 V for EC-69.

The calibration curve for fluorescent response is shown in Fig. 4. The linear fit is not a perfect approximation over a large interval [4], but in the region up to a temperature increase of $15^{\circ} \mathrm{C}$ the fit is adequate. Standard deviation increases for temperatures over $10^{\circ} \mathrm{C}$ and this is believed to be due to the fact that background intensity might vary during the long time measurements. A data acquisition sometimes required more than 20 minutes before the temperature on the platform was stabilized. When measuring temperature increase due to losses in transducers and without heating the whole device, higher accuracy is expected. In the interval of $1-10{ }^{\circ} \mathrm{C}$ temperature increase, the standard deviation is $0.67^{\circ} \mathrm{C}$.

The results of the temperature measurements is shown in Fig. 5, where a comparative measurement performed for the selected needle hydrophone conditions $\left(P_{i}\right.$ of $\left.3 \mathrm{Pas}^{1 / 2}\right)$ shows that the piezoelectrically harder material is more favorable when taking heating effects into account. EC-69 gives a lower temperature increase (relative room temperature) of $2.2{ }^{\circ} \mathrm{C}$ and $2.1{ }^{\circ} \mathrm{C}$ for serie and parallel resonance respectively compared to the higher temperature increase of $5.8{ }^{\circ} \mathrm{C}$ and $5.2{ }^{\circ} \mathrm{C}$ for the soft material (EC-76). The temperature curves for $\mathrm{EC}-76$ serie and parallel resonance is measured while the curve for EC-69 is predicted based on a 
measurement at $P_{i}$ of $3 \mathrm{Pas}^{1 / 2}$ and the assumption that the temperature increase relative EC-76 will be fix. Other measurements [5] predict anti-resonance frequency to be more favourable than resonance and this is seen for the piezoelectrically softer material (EC-76) but is not significant for the piezoelectrically harder material (EC-69). Since the slope of the curve increases for higher pressures, the relative temperature increase is larger at higher acoustic output pressure. An output pressure of $4 \mathrm{Pas}^{1 / 2}$ causes a temperature rise for series and resonance of EC-76 of $10.4{ }^{\circ} \mathrm{C}$ and $8.4{ }^{\circ} \mathrm{C}$ respectively. For EC-69 the predicted temperature is only 4 ${ }^{\circ} \mathrm{C}$.

The most important issue for the results in this study might be the reliability of the measurement method for the temperature increase in this application. The fluorescent response is very sensitive and therefore requires very good control of parameters that affect concentration of Rhodamine B. Especially sticking of Rhodamine B inside channel may be an issue. This was addressed by keeping time interval between a wash pulse and exposure fixed and within a time

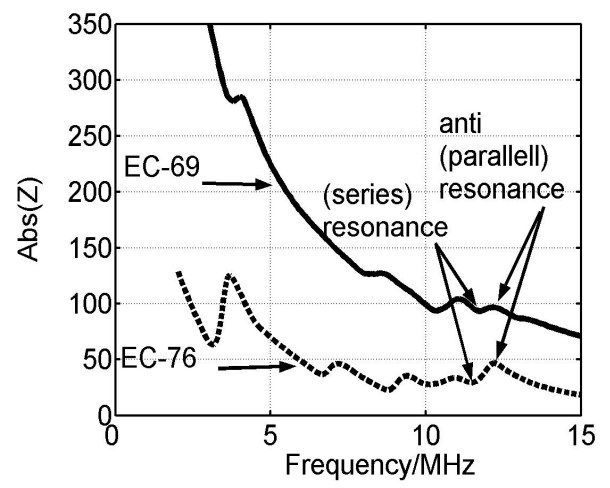

Figure 2. Impedance spectra with thickness resonances marked for soft (EC76) and hard (EC-69) PZT.

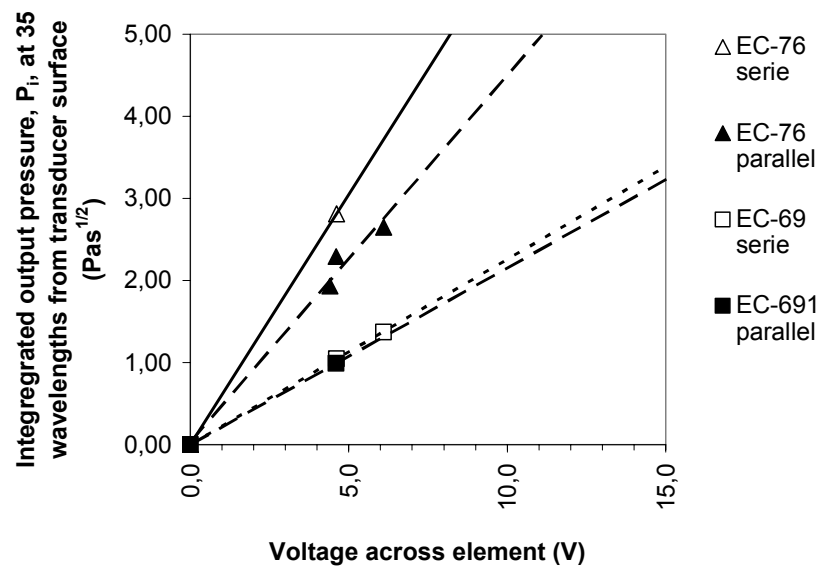

Figure 3. The variation of output pressure is measured with a needle hydrophone for parallel and series resonance for EC-76 and EC-69 at a distance of 35 wavelengths from the transducer.

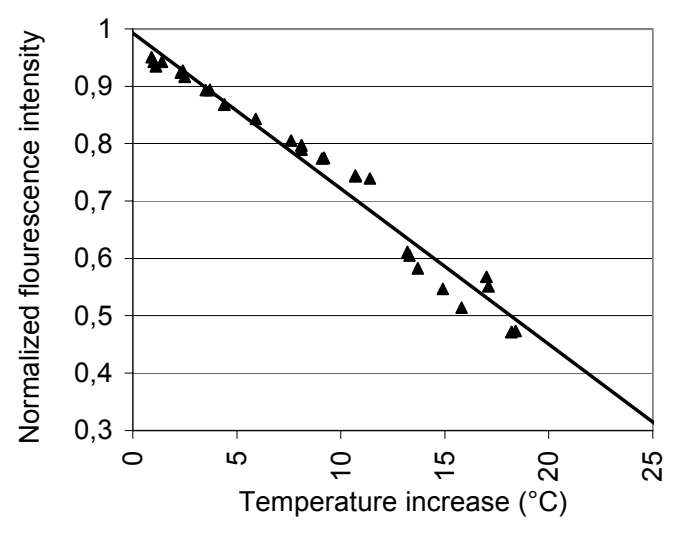

Figure 4. A calibration curve fit of fluorescence intensity decrease as a function of temperature increase.

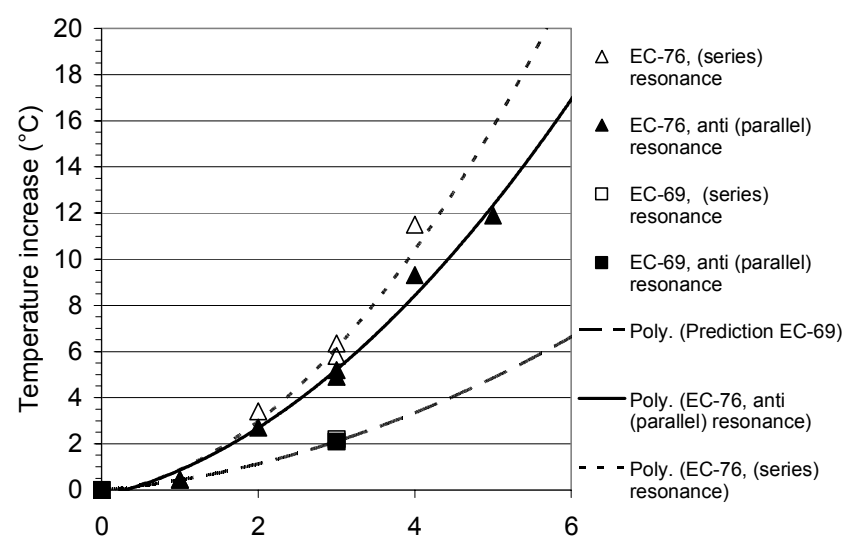

Integrated output pressure, $P_{i}$, at 35 wavelengths from transducer surface $\left(\mathrm{Pas}^{1 / 2}\right)$

Figure 5. Thermal measurements with Rhodamine B in a microchannel as a function of output acoustic pressure. For EC-69 the curve is predicted based on a similar relative temperature increase as a function of temperature as for EC-76 serie and parallel resonance.

frame of 2-10 minutes where the intensity was found to be repeatable and stable. During measurement of calibration curve for florescent response this effect is more prominent since stabilization of temperature in the whole device required much longer times. Sticking is believed to occur mainly at the bottom channel surface of epoxy and PZT and less at the silicon channel side and glass reflector channel top. A larger fluid flow might reduce the sticking, but flow also needs to be kept at a low enough level to maximize temperature rise in the fluid layer. In general the fluorescent measurement method requires very good control of the fluidics.

There are several experimental details that influence the results. One factor is the degree of polarization for the piezoelectrically harder PZT, which for these small transducers is hard to determine. Polarization of EC-69 is 
performed at $3 \mathrm{~V} / \mu \mathrm{m}$ and temperatures around $140{ }^{\circ} \mathrm{C}$. For these small transducers it is not possible to increase voltage higher without getting electrical breakdown. However, the piezoelectrically harder material showed superior performance even if complete polarization has not been confirmed. Another experimentally-related factor is the misalignment that may occur when positioning the needle hydrophone at the transducer surface. This misalignment is approximated to maximum $0.1 \mathrm{~mm}$ and is not believed to cause a large change in measured output pressure which is performed at a distance of more than $4 \mathrm{~cm}$. A third factor is that the impedance spectrum has been measured at low voltages $(1.6 \mathrm{~V})$. At these voltages, no significant change in impedance spectra between water-filled channels and air is observed. Measurements at higher voltages would conclude if resonance frequencies and in water-filled channels stay unchanged for higher drive voltages.

There are also several other factors that affect the temperature measurements. The assembly of transducers gives small misalignments with regards to the reflecting surface and this could affect the temperature measurements. Also, channel height is matched for a resonance frequency of 12.2 MHz. The resonance frequencies tested differ somewhat in degree of matching to the channel height, and the cavity resonance efficiency might therefore vary slightly. However, these geometry related effects are believed to be relatively small.
Additional measurements to confirm temperature-pressurecurve for EC-69 will be performed in the future.

\section{CONCLUSIONS}

The overall comparison of temperature increase for EC-76 and EC-69 for parallel and series resonance show that the piezoelectrically harder material is more favourable from a temperature point of view, and that it enables trapping in our microfluidic platform with only a few degrees of temperature increase. The accuracy in the measurements we believe is limited by the effect of sticking of Rhodamine $\mathrm{B}$ at channel walls. However, in the range of $1-10{ }^{\circ} \mathrm{C}$ temperature increase, the standard deviation of the calibration curve is reasonable low $\left(0.67^{\circ} \mathrm{C}\right)$.

\section{REFERENCES}

1. D. Bazou, L.A. Kuznetsova, W.T Coakley, Physical enviroment of 2-D animal cell aggregates formed in a short pathlength ultrasound standing wave trap, Ultrasound in Medicine and Biology, 31, 423, (2005)

2. T. Lilliehorn, U. Simu, M. Nilsson, M. Almqvist, T. Stepinski, T. Laurell, J. Nilsson, S. Johansson, Trapping of microparticles in the near field of an ultrasonic transducer, Ultrasonics, 43, 293, (2005)

3. M. Nilsson et al, Acoustic manipulation of cells in a microfluidic format, to be presented at $\mu$ TAS-2005

4. D. Ross, M. Gaitan, and L. E. Locascio, Temperature Measurement in a Microfluidic Systems using a Temperature-Dependent Fluorescent Dye, Analytical Chemistry, 73, 4117, (2001)

5. K. Uchino, J. Zheng et al., High Power Characterization of Piezoelectric Materials, J. Electroceramics 2, 1, 33-40, 1998 